\title{
New recognition of hepatic perivascular epithelioid cell tumours (PEComa): a retrospective analysis of 35 cases
}

\author{
Xiao Yang \\ Nanjing University Medical School \\ Qing Yuan Wang \\ Nanjing University Medical School \\ Xiao Zhou \\ Nanjing University Medical School \\ Haoming Zhou \\ Nanjing University Medical School \\ Wen Bo Jia \\ Nanjing University Medical School \\ Chun Yang Hu \\ Nanjing University Medical School \\ Jian Chu \\ Nanjing University Medical School \\ Lian Bao Kong ( $\square$ lbkong@njmu.edu.cn ) \\ Nanjing University Medical School
}

\section{Research}

Keywords: Hepatic PEComa; Clinical features; Diagnosis; Treatment; Prognosis

Posted Date: February 6th, 2020

DOl: https://doi.org/10.21203/rs.2.22805/v1

License: (c) (1) This work is licensed under a Creative Commons Attribution 4.0 International License. Read Full License 


\section{Abstract}

Purpose Our primary objective was to investigate the clinical features, diagnosis, treatment and prognosis of hepatic perivascular epithelioid cell tumours (PEComa).

Patients and Methods Thirty-five cases of pathologically proven hepatic PEComa that were treated in the Department of Hepatobiliary Center of the First Affiliated Hospital of Nanjing Medical University from January 2008 to February 2019 were retrospectively analysed, and the literature was reviewed.

Results Twenty-nine females and 6 males were included in this study. The mean age of these patients was 48.0 years (range, 21-75 years). Thirteen patients complained of upper abdominal pain and discomfort, but the tumours in the other patients were identified by performing an imaging examination. Hepatic PEComas tended to occur in the right lobe of the liver (20 cases in the right lobe, 13 in the left lobe and 2 in the caudate lobe). Two cases were characterized by multiple tumours, and the remaining cases were single lesions (range, 1.2-12 cm). Only 8 cases were correctly diagnosed by the preoperative imaging examination, and the correct diagnosis rate was only $22.9 \%$. The postoperative immunohistochemistry analysis showed that hepatic PEComas are positive for human melanoma black 45 (HMB-45), Melan-A and smooth muscle actin (SMA), with the exception of 1 case that was negative for Melan-A. All patients undergoing an operation accepted regular follow-up, and the average time was 66.5 months (range, 3-132 months). Two patients who experienced tumour recurrence and 1 patient who died due to cardiovascular disease, but the remaining patients showed no evidence of tumour recurrence or metastasis during the follow-up period.

Conclusions Hepatic PEComa is a rare type of tumour that mainly occurs in young and middle-aged women. The lack of clinical manifestations and imaging findings increases the difficulty of determining a preoperative diagnosis, which mainly depends on the pathological examinations. Surgery is currently the only effective treatment, and long-term clinical follow-up is necessary due to the aggressive behaviour and relapse of hepatic PEComa in some cases.

\section{Introduction}

Hepatic perivascular epithelioid cell tumours (PEComa) specifically express markers of melanocytes and myocytes when examined using immunohistochemistry and are a type of rare neoplasm originating from mesenchymal tissues [1]. In 1996, Zamboni et al. formally classified a group of related mesenchymal tumours as "PEComa", a term that was first proposed by Bonetti in 1992 [2]. The World Health Organization (WHO) officially described PEComas as "a kind of abnormal mesenchymal tumour consisting of perivascular epithelioid cells with unique histological and immunohistochemical features" [3]. However, the origin and biological behaviours of PEComa remain unclear. PEComas are usually divided into two types. The first is a special group containing four types: pulmonary clear cell "sugar" tumour (CCST), clear cell myomelanocytic tumour of the falciform ligament/round ligament (CCMMT), lymphangioleiomyomatosis (LAM) and angiomyolipoma (AML). The second is a non-special type called 
PEComa-not otherwise specified (PEComa-NOS) and occurs in the abdomen, pelvis, digestive tract and other tissues with a low incidence; thus, it has no formal name [4].

Hepatic PEComas are benign tumours with some malignant biological behaviours. Although hepatic PEComas are extremely rare, the incidence has increased in recent years and some malignant biological behaviours are potential threats to people [2]. On the one hand, we have little knowledge of the clinical features, imaging findings and pathological characteristics of hepatic PEComas. On the other hand, the cases reported in the literature are limited. More samples must be collected to explore their clinical manifestations, diagnostic methods and treatments and to increase the awareness of this disease [5]. We retrospectively analysed the clinical features, diagnosis, treatment and prognosis of 35 patients with hepatic PEComa in our single centre. After consulting the literature, the number of cases of hepatic PEComa reported in our single centre was the largest.

\section{Patients And Methods}

\subsection{Patients}

We collected data from 35 patients with hepatic PEComa who were treated in the Department of Hepatobiliary Center, the First Affiliated Hospital of Nanjing Medical University from January 2008 to February 2019. The inclusion criteria for patients in this study were patients were diagnosed with hepatic PEComa for the first time, as confirmed by a routine pathological and immunohistochemical diagnosis after the operation, as well as patients who had not received any related preoperative therapy before hospitalization. People who suffered from malignant tumours or other liver neoplasms were excluded from this study.

Every patient received laboratory examinations, including routine blood analyses, analyses of liver function, and analyses of hepatitis virus and tumour-specific markers, before the operation. Twenty-eight patients underwent a computed tomography (CT) examination, 21 patients received ultrasonography, and 8 patients were examined with magnetic resonance imaging (MRI) upon admission. Furthermore, all patients were treated with partial hepatectomy. With the exception of 1 case for which only routine pathological examination results were available, pathological and immunohistochemical examinations were performed on each tumour specimen section. This study was approved by the institutional ethical review board, and written informed consent was provided by all patients and their guardians.

\subsection{Preoperative features}

Clinical data for all patients were adequately recorded. First, the age, sex and course of disease of every patient were included in the statistical analysis, which also contained the clinical manifestations and positive signs. Second, the risk factors that were possibly associated with the onset of hepatic PEComa, including smoking, drinking, hypertension, diabetes, hepatitis, use of contraceptive drugs, history of surgery and hereditary history, were considered in this study. Finally, the results of laboratory and imaging examinations of all patients were also included and analysed. 


\subsection{Therapeutic methods}

Every patient accepted a partial hepatectomy under general anaesthesia and tracheal intubation, and the tumours were dissected with negative surgical margins. After the first hepatic hilum was blocked during the operation, the clamp method was used to resect the tumour together with $1 \mathrm{~cm}$ of the surrounding normal liver tissue. No patient received chemotherapy, radiotherapy or other treatments after surgery. Long-term follow-up and periodic review were conducted simultaneously after discharge.

\subsection{Postoperative pathology}

The tumour specimens were routinely fixed with $4 \%$ formaldehyde, embedded in paraffin and then sectioned. Haematoxylin-eosin (HE) staining and immunohistochemical EnVision staining were performed on the sections. A group of antibodies, including Melan-A, human melanoma black 45 (HMB45), smooth muscle actin (SMA), vimentin (Vim), CD34, CD117, E-cadherin (E-cad), Ki-67, CK8, CK18, hepatocyte (Hep), alpha-fetoprotein (AFP) and S100, were used for immunohistochemistry. At least two pathologists diagnosed all specimens.

\subsection{Follow-up}

All patients were followed by telephone or outpatient review, and the follow-up period extended up to 28th February 2019. Outpatient review was mainly based on liver function and ultrasonography or CT examinations to determine whether the tumour has recurred or metastasized. The telephone follow-up was performed to understand the patients' physical conditions and quality of life. One patient died during the follow-up period due to cardiovascular disease, but all other patients survived.

\section{Results}

\subsection{Clinical history and presentation}

Twenty-nine females and 6 males were included in this study; thus, the male-to-female ratio was 1:4.8. The mean age of these patients was 48.0 years (range, $21-75$ years). According to the age division stipulated by the WHO, 23 female patients were aged less than 60 years (young and middle-aged women), accounting for $65.7 \%$ of the total number. Hepatic PEComas are more likely to occur in young and middle-aged females. Thirteen patients presented with upper abdominal intermittent pain or discomfort, while 22 patients presented a hepatic mass upon an imaging examination. One patient experienced a fever due to choledocholithiasis, and positive signs of tenderness in the liver region existed in 9 patients (Table 1$)$.

In this study, only 1 person had a history of smoking and drinking. Six patients had a history of hypertension, while 1 patient had a history of diabetes. Notably, 7 female patients had previously received a gynaecological operation. Furthermore, none of the cases were found associated with tuberous sclerosis complex (TSC) or contraceptive drugs. Similarly, the medical history and lifestyle of every patient appeared to be unrelated to the occurrence of hepatic PEComa. With the exception of two patients 
who presented with positive hepatitis B surface antigen, routine blood analyses, liver functions, hepatitis virus and specific tumour markers, including AFP, carcinoembryonic antigen (CEA) and carbohydrate antigen $19-9$ (CA19-9) levels, remained within the normal ranges.

\subsection{Imaging findings}

Hepatic PEComa tended to occur in the right lobe of the liver (20 cases in the right lobe, 13 in the left lobe and 2 in the caudate lobe). Two patients with multiple tumours, and the other patients presented single lesions. The mean size of tumours was $4.8 \mathrm{~cm}$ (range, 1.2-12 cm). Thirteen tumours were larger than $5 \mathrm{~cm}$. Nine tumours were unable to be diagnosed, and 10 were misdiagnosed as hepatocellular carcinoma (HCC). Only 8 cases were diagnosed correctly, and other tumours were regarded as lipoma, hamartoma, hepatic haemangioma, intrahepatic cholangiocarcinoma (ICC) or focal nodular hyperplasia $(\mathrm{FNH})$. The correct diagnosis rate of preoperative imaging examinations was only $22.9 \%$.

Ultrasonography was performed on 21 patients before surgery and all of these tumours were a heterogeneous mass with a high echogenicity. The form of hepatic PEComa with a clear boundary was irregular. However, 3 tumours presented low echogenicity on ultrasound images (Fig. 1). Colour Doppler flow imaging (CDFI) identified rich intralesional vascularization. The imaging findings of hepatic PEComa with high echogenicity on ultrasound were limited and similar to hepatic haemangioma. Therefore, 7 patients were initially misdiagnosed with hepatic haemangioma, and thus, ultrasound is not appropriate as the sole modality for diagnosis. Then, 28 patients underwent a CT examination, and 8 were examined with MRI upon admission. The plain CT scan exhibited the tumour as a patchy or quasi-circular mass with an inhomogeneous low density and unclear or clear boundaries. In both the arterial phase and portal phase, the lesions displayed obvious heterogeneous enhancement. The enhancement of lesions was significantly weakened in the venous phase, and the neoplasm decayed to a low-signal state in the delayed phase (Fig. 2). Using a combination of CT and MRI, 10 cases of lesions containing lipid components were identified. Haemorrhaging, cystic degeneration, necrosis or calcification was not observed.

MRI revealed heterogeneous hypointensity on T1-weighted images ( $\mathrm{T} 1 \mathrm{WI})$ and hyperintensity on $\mathrm{T} 2$ weigthed images (T2WI) of 7 specimens. Only 1 tumour displayed hyperintensity both on T1WI and T2WI. Hepatic PEComas appeared as high signal masses on diffusion-weighted imaging (DWI), whereas they showed the opposite signal on apparent diffusion coefficient (ADC) images (Fig. 3). The findings of enhanced MRI were consistent with the CT data.

\subsection{Pathological features}

\subsubsection{Gross examination}

The tumours with a round or quasi-circular shape were all circumscribed, and most were soft or moderate in texture. However, 3 masses were hard with poor activity. With the exception of 1 tumour characterized by invasive growth, the tumours showed an exophytic growth pattern. Thirty-two tumours were clearly demarcated from the surrounding tissues, while the borders were not clear in the other 3 cases. The cut 
surface of tumours was light yellow, off-white, pinkish-grey or dark red, and a complete capsule was observed in 7 tumours. Interestingly, the appearances of the tumour sections were diverse, as 4 tumours exhibited a haemangiomatous appearance and 3 neoplasms displayed a fleshy or variegated appearance. When 2 cases of double lesions were included, the largest tumour was $12 \mathrm{~cm}$ in diameter (Table 2).

\subsubsection{Microscopic detection and immunohistochemistry}

Hepatic PEComa was mainly composed of three types of cells, epithelioid cells, spindle cells and eosinophilic cells, which were arranged radially around abnormally dilated blood vessels. In 4 cases, epithelioid cells were predominant, while the others contained a mixture of the cell types. In addition, thick-walled blood vessels, 1 tumour with necrosis, 4 tumours megakaryocytes and mild cell atypia, 9 tumours with abundant dilated hepatic sinusoids and 32 tumours with mature adipose tissues were also observed. The tumour cells were arranged in sheets or nests. Epithelioid cells with a transparent and vacuolar cytoplasm were polygonal or round in shape and uniform in size. However, a few cells with a red cytoplasm and clear periphery displayed a spider-like morphology. Spindle cells with a red-stained cytoplasm were analogous to smooth muscle cells. Light red eosinophilic granules were observed in the cytoplasm of eosinophilic cells and the nuclei were slightly atypical (Fig. 4).

Except for 1 case without an immunohistochemical report, the immunohistochemical staining of hepatic PEComas was positive for HMB-45 and SMA in all 34 cases, for Melan-A in 33 cases, for E-cad in 9 cases, for CD34 in 7 cases, for S-100 in 3 cases and for Vim in 2 cases (Fig. 5). Additionally, the tumour cells were negative for AFP, CK8, CK18, CD117 and Hep. Except for 1 tumour in which Ki-67 staining reached $25 \%$, the Ki-67 staining was less than $10 \%$ in all tumours. All neoplasms tended to be benign, and no residual tumour cells were detected in the incision margins.

\subsection{Treatment and follow-up}

Partial hepatectomy was performed on every patient, and complete resection of tumours was guaranteed during operation. All patients were discharged smoothly after the operation and no deaths occurred during perioperative period. Patients were regularly reviewed by B-mode ultrasound or CT annually without accepting any other treatments, and the average follow-up period was 66.5 months (range, 3-132 months). During the follow-up period, the tumours recurred in 2 patients, who then received secondary resection in our hospital. In addition, 1 patient died due to cardiovascular disease, and the remaining patients showed no signs of tumour recurrence or metastasis at the end of follow-up.

\section{Discussion}

PEComas mainly develop in the kidney and uterus, while the occurrence of PEComa is very rare in the liver [6]. Yamasaki et al. [7] first reported a case of hepatic PEComa that occurred in a 30-year-old woman. More than 200 cases of PEComa have been reported to date, but most of them were AML [8]. In fact, the definition of PEComa reported in many studies refers to the non-special type of the PEComa family called PEComa-NOS, which was named as epithelioid angiomyolipoma or perivascular epithelioid cell tumour 
[9]. However, due to its very low incidence, a unified scientific definition of these tumours is not available. In 2013, the WHO unified the names of these tumours, including AMLs and PEComa-NOS. Despite the controversy, the concept of PEComa has gradually been used instead of these original names [10]. With the increasing number of reports of hepatic PEComa, physicians have gradually improved their understanding of this tumour. However, due to its rarity, the origin and diagnostic criteria for this tumour have not yet been clarified [11].

A PEComa can occur in many parts of the human body, such as the uterus, skin, thorax, gastrointestinal tract and liver. The prognosis of benign hepatic PEComas, which are able to be radically cured by surgery, is usually satisfactory and little evidence of recurrence or metastasis of these tumours has been reported after surgery in previous case studies [1-11]. In contrast, malignant hepatic PEComas characterized by aggressive growth and early metastasis have a poor prognosis, but the incidence is much lower than benign hepatic PEComas [12]. Studies have confirmed that $6-10 \%$ of cases of hepatic PEComa are associated with a genetic disease called TSC, because the pathogenesis of PEComa may be related to the deletion of TSC1 (9q34) or TSC2 (16p13.3) [2]. Shih-Chiang Huang et al. [13], who had analysed specimens of hepatic PEComa, also found that hepatic PEComas are associated with an alteration in the mTOR pathway and a loss of heterozygosity (LOH) in the TSC1/TSC2 genes, particularly in TSC2. However, in our study, none of the 35 patients with hepatic PEComa had a history of similar hereditary diseases in their family. Further studies are needed to determine whether the aetiology of hepatic PEComa is related to other factors.

Based on the results, hepatic PEComa tends to occur in young and middle-aged females, who accounted for $65.7 \%$ of patients in our study, with an average age of 48.0 years. This finding was consistent with the results of statistical analyses reported in previous studies [3][8][14]. Most of the tumours were identified accidentally in patients without obvious clinical symptoms or manifestations during a physical examination. Nevertheless, when the tumours grow so large that they will compress the surrounding tissues, patients may experience signs of tenderness in the liver region and suffer from upper abdominal pain or discomfort with gastrointestinal reactions such as nausea and vomiting [15]. In general, the lack of specific clinical manifestations makes this tumour difficult to diagnose, but the signs and symptoms caused by hepatic PEComa potentially indicate the sizes and locations of tumours. No evidence was available to prove that the risk factors smoking, alcoholism, hepatitis, contraceptive drug use, surgical history and chronic diseases are associated with hepatic PEComa in this study. However, hormone levels may play an important role in the occurrence of hepatic PEComa [8]. In terms of laboratory examinations, all the indexes of patients were normal, indicating that this tumour is difficult to diagnose based on a laboratory examination alone because of the lack of specific tumour markers.

An imaging examination is an important method to diagnose hepatic PEComa before an operation, but the accuracy of the imaging diagnosis was unsatisfactory due to the different components of tumours and a wide variety of patterns [16]. The absence of adipose tissues and vascular components in some tumours is another important explanation for the misdiagnosis [17]. Even when CT and MRI data were combined, the rate of an accurate diagnosis was still only $22.9 \%$, similar to the rate of $20 \%$ reported in 
other studies [18][19]. According to the results of this study, hepatic PEComas were more likely to occur in the right lobe. However, combined with the findings from other studies, as the sample size increases, the number of tumours occurring in the left lobes was approximately equal to the right [1-19].

Many specific manifestations of hepatic PEComa are able to be observed using CT and MRI, which will help effectively improve the diagnostic accuracy. First, a circular or quasi-circular inhomogeneous occupancy with a low density, clear boundaries and expansive growth has been observed on a plain scan, and adipose tissue, haemorrhage, necrosis and cystic degeneration have occasionally been detected in the tumours [4]. Second, the lesions with the features of neovascularization and arteriovenous connections are heterogeneously enhanced obviously in the arterial phase, which is the most important condition for the diagnosis of a hepatic PEComa [19]. Additionally, abnormal enhancement of enlarged blood vessels in the edge or centre of tumours is another significant characteristic of hepatic PEComas [20]. In the portal phase, the enhancement persists, and it will be significantly weakened in the venous phase. If fewer blood vessels and more fatty components are present in tumours, they will exhibit features of low-density lesions in the delayed phase [21]. Hepatic PEComas have been confused with $\mathrm{HCC}$, FNH, lipoma, adenoma and hamartoma on a CT scan [16]. In particular, the imaging findings of highly differentiated HCC are very similar to fat-poor hepatic PEComas, and thus investigators have proposed some suggestions to help distinguish them. 1. HCCs complicated with necrosis, haemorrhage and cystic degeneration are common and do not contain adipose tissues. 2. Although HCC has the characteristic of "fast in and fast out", its internal vessels are usually small and accompanied by bleeding, which are different from the enlarged vascular shadows of hepatic PEComas. 3. HCC always presents aggressive growth and unclear boundaries, and is generally accompanied by cirrhosis. Larger hepatocellular carcinomas tend to invade the portal vein and form a cancer thrombus [3] [20-21]. After mastering these characteristics, the tumour is not difficult to diagnose when the findings are combined with the history of hepatitis and an increase in the serum AFP level.

In the present study, hepatic PEComas presented as a heterogeneous hypointensity on T1WI and hyperintensity on T2WI. However, as the cystic components decrease and the substantial parts increase, the tumour will be hyperintense on T1WI [22]. Hepatic PEComas are viewed as high signal masses on DWI, whereas on ADC, they may present the results. In some larger tumours, delayed enhancement of pseudocapsule is observed in the delayed phase of MRI [23]. Although none of the cases in this study was diagnosed using B-mode ultrasonography, ultrasound is still capable of showing whether the tumours have involved the surrounding tissues, and CDFI can discover the amount of blood supply in tumours, which is helpful to differentiate hepatic PEComas from other lesions. Using the contrast agent called Sonazoid, B-mode ultrasonography can also determine the significant enhancement of tumours in early stage and rapid excretion of contrast agent [24]. Larger hepatic PEComas display a radioactive concentration on positron emission tomography (PET)/CT imaging, which is different from most benign liver tumours [25] [26]. The combination of various imaging examinations will improve the accuracy of diagnosis to a certain extent. 
As a type of abnormal mesenchymal tumours consisting of perivascular epithelioid cells, which are positive for both myogenic marker (SMA) and melanogenesis markers (HMB-45 and melan-A), the origin of hepatic PEComas remains uncertain. However, some scholars have suggested that PEComa may originate from undifferentiated neural crest cells with the two phenotypes described above [21]. In additions, PEComa may stem from myoblasts or smooth muscle cells that have mutated at the molecular level, and the pericytes are likely to be the ancestors of PEComa [27]. The volume of a hepatic PEComa can change over a wide range. Maebayashi et al. [3] examined 74 cases and reported a maximum size of these tumours of $25 \mathrm{~cm}$, and the minimum size was $1.2 \mathrm{~cm}$ in our study. Although hepatic PEComa is typically benign, the potential for malignant biological behaviours, such as aggressive growth, haemorrhage, necrosis and rupture, still exist [15] [28].

Typical hepatic PEComas consist of mature adipose tissues and thick-walled or thin-walled blood vessels around which the polygonal or round epithelioid tumour cells are radially arranged [29]. In some cases, vascular hyalinization and spindle cells resembling smooth muscle cells have been observed [29]. In fact, the tumour cells also contain other cell types, including vacuolar cells with a transparent cytoplasm and eosinophilic cells with atypical nuclei. Another important feature is that abundant dilated hepatic sinusoids and extramedullary haematopoiesis have been observed in some cases [10]. Although many cases of hepatic PEComa have been reported, definite criteria for the antidiastole on benign and malignant hepatic PEComa have not been developed. Folpe et al. [30] first proposed the diagnostic criteria for potential malignant PEComas, including a tumour size $>5 \mathrm{~cm}$, infiltration into surrounding tissues, vascular involvement, high nuclear grade, high cellularity, necrosis and a mitotic figure $>1 / 50$ high power field (HPF). If more than 2 conditions are met, the tumours are potentially malignant.

According to this standard, 10 potentially malignant tumours were identified in our study, while recurrence of the neoplasm only occurred in 1 patient. In contrast, the neoplasm was considered a benign tumour and reappeared after surgery. Fortunately, the 2 patients have survived for more than two years after the secondary operation without recurrence. Combined with recent reports, there are still some limitations in this view presented by Folpe. Jafari et al. [31] showed that even if the above conditions were not met, there were two cases of hepatic PEComa reported to exhibit malignant biological behaviours that caused fatal consequences, such as invasive growth patterns and distant metastasis. In contrast, some tumours with potential malignancy may have a good prognosis. Nuclear atypia and multinucleated giant cells can also appear in benign hepatic PEComas. The aggressive behaviours, larger tumour size $(>10 \mathrm{~cm})$ and coagulative necrosis are essential features to diagnose malignant hepatic PEComas [2]. More detailed diagnostic criteria must be developed.

In this study, only 1 tumour was negative for Melan-A, and all hepatic PEComas displayed positive immunohistochemical staining for HMB-45 and SMA. Thus, the expression of Melan-A, HMB45, and SMA are the most important evidence for hepatic PEComa. This result was consistent with other studies [131 ]. Surprisingly, these markers were not $100 \%$ positive in previously reported cases, and Folpe et al. [30] found that only $80 \%$ of PEComas were simultaneously positive for melanocyte and smooth muscle cell markers. When the expression of SMA or melan-A is negative, the diagnosis of hepatic PEComa cannot be excluded. HMB-45 is expressed at higher levels than SMA when epithelioid cells are the predominant 
cell type in tumours, while the opposite result is observed when spindle cells are the predominant cell type [15]. In addition to the markers described above, the phenotype of tumour cells will vary with the changes in the neoplastic composition. The finding of $9 \mathrm{E}$-cad-positive tumours $d$ and 7 CD34-positive tumours in our study has not been described in other studies [1-31]. The expression of CD34 may be related to extramedullary haematopoiesis and an increase in the vascular component. E-cad may be related to the morphological changes in tumour cells, and S-100 is expressed by adipocytes. Furthermore, hepatic PEComas are generally negative for CD117, AFP, Hep and CK8/18 and positive for Vim, microphthalmiaassociated transcription factor (MITF) and S-100 [1-31]. Transcriptional enhancer factor-3 (TEF-3) and $\mathrm{Ki}-67$ help clinicians judge the degree of malignancy of hepatic PEComa. A hepatic PEComa in which greater than $20 \%$ of cells express Ki-67 or displaying high levels of TEF expression may be malignant [15] [32].

The diagnosis of hepatic PEComa mainly relies on the unique morphology of tumour cells and presence of specific immunological markers including HMB-45, SMA and Melan-A. Fine-needle biopsy (FNB) is recommended for the diagnosis of hepatic PEComa, which effectively avoids the substantial trauma caused by surgery [22]. In additions, hepatic PEComas must be differentiated from other tumours. First, epithelioid tumours such as HCC and metastatic clear cell carcinoma are easily confused with hepatic PEComas in terms of morphology, but they are all negative for melanocytic markers (HMB-45 and MelanA). Second, soft tissue clear cell sarcoma and malignant melanoma, which may express HMB-45 and Melan-A, also deserve attention. However, compared with hepatic PEComas, these tumour cells are more atypical and do not express myogenic markers (SMA).

The majority of hepatic PEComas are benign and usually have a good prognosis. A few cases of recurrence or distant metastasis of benign hepatic PEComas have been reported after surgery, but the malignant hepatic PEComas generally display the opposite behaviours. Malignant tumours often metastasize early through intraperitoneal implantation and haematogenous metastasis. Cases with lymph node metastasis have not yet been reported [33]. Five of 7 patients with malignant hepatic PEComa reported died after surgery in a previous study, and the median survival time was only 7 months [33]. In the study by Simon et al. [34], five patients with unresectable malignant PEComas were treated with adjuvant chemotherapy, but 4 tumours were fatal. In our study, all 35 tumours were benign according to the pathological findings and clinical evidence, despite the potential for malignancy in 10 cases. The tumour recurred in two cases, and 1 patient died of cardiovascular disease, which was not related to the hepatic PEComa, during the follow-up period. In these two patients, the recurrence occurred more than 3 years after operation. The possibility of long-term recurrence still exists, even in patients with benign hepatic PEComas, and long-term follow-up is necessary for patients.

A systematic therapeutic schedule has not been determined, but surgical resection is recognized as an ideal approach to cure hepatic PEComa. The vast majority of benign PEComas will not recur after surgical excision and the prognosis is good. The key goal of the operation is to ensure the complete removal of tumours and avoid dissemination caused by tumour rupture [32]. However, malignant hepatic PEComas lack effective treatments, and patients who have received operation may experience tumour 
recurrence and distant metastasis. Additionally, these tumours are not sensitive to conventional radiotherapy and chemotherapy [15] [34]. However, many therapeutic methods have been used to treat these tumours. Francesca et al. [5] adopted neoadjuvant sirolimus therapy as a treatment for a large malignant hepatic PEComa to reduce its size prior to the operation. After surgery, the patient accepted neoadjuvant sirolimus therapy for an additional 6 months. Although the therapeutic efficacy remains unclear, sirolimus actually inhibits the growth of tumours. Because tumourigenesis is related to the activation of the mTOR pathway, sirolimus, which inhibits the mTOR pathway, is effective against malignant hepatic PEComas [35]. Stereotactic body radiation therapy (SBRT) has also been used to prevent the recurrence and metastasis of malignant hepatic PEComas after surgery [34]. These methods represent experimental treatments and their therapeutic effects require further evaluation and research.

\section{Conclusions}

Hepatic PEComas are a rare type of tumours that mainly occur in young and middle-aged women. The lack of clinical manifestations and imaging findings increases the difficulty of establishing a preoperative diagnosis, which mainly depends on the results of pathological examinations. Surgery is currently the only effective treatment, and long-term clinical follow-up is necessary to monitor the aggressive behaviour and relapse of hepatic PEComa in some patients.

\section{Declarations}

\section{Ethics Statement}

This retrospective study and the publication of clinical data were approved by the Ethics Committee of Nanjing Medical University. Written informed consent was also provided by the patients.

\section{Disclosure Statement}

None of the authors has competing interests to declare.

\section{Funding Sources}

No funding was received for this study.

\section{Authors' Contributions}

Xiao Yang, Lianbao Kong and Haoming Zhou designed and performed this retrospective study. Xiao Zhou, Jian Chu, and Chunyang Hu collected the clinical data. Wenbo Jia analysed the clinical data. Xiao Yang, Qingyuan Wang and Xiao Zhou wrote the paper. Haoming Zhou and Lianbao Kong revised the article.

\section{References}


[1] Hornick JL, Fletcher CD. PEComa: what do we know so far. Histopathology. 2006; 48 (1) :75-82.

[2] Liu Z, Qi Y, Wang C, Zhang X, Wang B. Hepatic perivascular epithelioid cell tumor: Five case reports and literature review. Asian J Surg. 2015;38(1):58-63.

[3] Maebayashi T, Abe K, Aizawa T, Sakaguchi M, Ishibashi N, Abe O, et al. Improving recognition of hepatic perivascular epithelioid cell tumor: Case report and literature review. World J Gastroenterol. 2015;21(17):5432-5441.

[4] Chen MD, Wang L, Li Q, Tan Y, Wang YC, Ma HF. CT and MRI features of hepatic perivascular epithelioid cell tumor. J Med Imaging. 2018;28(06):967-970.

[5] Bergamo F, Maruzzo M, Basso U, Montesco MC, Zagonel V, Gringeri E, et al. Neoadjuvant sirolimus for a large hepatic perivascular epithelioid cell tumor (PEComa). World J Surg Oncol. 2014;12(1): 46-49.

[6] Zhan CN, Liu DR, Shi DK, Xu YL. Hepatic perivascular epithelioid cell tumor (PEComa): report of a case and literature review. Chin J Hepat Surg (Electronic Edition). 2014;3(05):31-34.

[7] Yamasaki S, Tanaka S, Fujii H, Matsumoto T, Okuda C, Watanabe G, et al. Monotypic epithelioid angiomyolipoma of the liver. Histopathology. 2000;36(5): 451- 456.

[8] Wang GY, Zhao C, Hou DF, Yang MW, Hu XR, Liu ZH, et al. Clinical analysis of hepatic perivascular epithelioid cell neoplasm: a report of eleven cases. Natl Med J China. 2018;98(34): 2715-2717.

[9] Ameurtesse H, Chbani L, Bennani A, Toughrai I, Beggui N, Kamaoui I, et al. Primary perivascular epithelioid cell tumor of the liver: new case report and literature review. Diagn Pathol.2014;9(1):149-155.

[10] Son HJ, Kang DW, Kim JH, Han HY, Lee MK. Hepatic perivascular epithelioid cell tumor (PEComa): a case report with a review of literatures. Clin Mol Hepatol.2017;23(1):80-86.

[11] Wang ZS, Xu L, Ma L, Song MQ, Wu LQ, Zhou X. Hepatic falciform ligament clear cell myomelanocytic tumor: A case report and a comprehensive review of the literature on perivascular epithelioid cell tumors. BMC Cancer.2015;15(1):1004-1012.

[12] Abhirup B, Kaushal K, Sanket M, Ganesh N. Malignant hepatic perivascular epithelioid cell tumor (PEComa) - Case report and a brief review. J Egypt Natl Canc Inst. 2015;27(4):239-242.

[13] Huang SC, Chuang HC, Chen TD, Chi CL, Ng KF, Yeh TS, et al. Alterations of the mTOR pathway in hepatic angiomyolipoma with emphasis on the epithelioid variant and loss of heterogeneity of TSC1/TSC2. Histopathology.2015;66(5):695-705.

[14] Tang D, Wang J, Tian Y, Li Q, Yan H, Wang B, et al. Hepatic perivascular epithelioid cell tumor: Case report and brief literature review. Medicine.2016;95(51): e5572. 
[15] Gao WK, Dai ZL. Hepatic perivascular epithelioid cell tumor (PEComa): a case report and literature review. J Clin Hepatol.2018;34(01):172-176.

[16] Tan Y, Xiao EH. Hepatic perivascular epithelioid cell tumor (PEComa): dynamic CT, MRI, ultrasonography, and pathologic features-analysis of 7 cases and review of the literature. Abdom Imaging. 2012;37(5):781-787.

[17] Jeon TY, Kim SH, Lim HK, Lee WJ. Assessment of triple-phase CT findings for the differentiation of fat-deficient hepatic angiomyolipoma from hepatocellular carcinoma in non-cirrhotic liver. Eur $\mathrm{J}$ Radiol.2010;73(3):601-606.

[18] Yang X, Li A, Wu M. Hepatic angiomyolipoma: clinical, imaging and pathological features in 178 cases. Med Oncol. 2013;30(1):416-421.

[19] Lee SJ, Kim SY, Kim KW, Kim JH, Kim HJ, Lee MG, et al. Hepatic angiomyolipoma versus hepatocellular carcinoma in the noncirrhotic liver on gadoxetic acid-enhanced MRI: a diagnostic challenge. AJR Am J Roentgenol. 2016; 207(3):562-570.

[20] Zhao XL, Hu RH, Wang HP, Chen ZY. CT and MRI manifestations of hepatic perivascular epithelioid cell tumor. Chin J Med Imaging. 2016;24(09):669-673.

[21] He YQ, Yao JJ, Liu JB, Li HL. MSCT manifestations of hepatic fat-poor perivascular epithelioid cell tumor. J Pract Radiol.2018;34(6):901-904.

[22] Khan HM, Katz SC, Libbey NP, Somasundar PS. Hepatic PEComa: a potential pitfall in the evaluation of hepatic neoplasms. BMJ Case Rep.2014; 6:2014-2019.

[23] Lee SJ, Kim SY, Kim KW, Shin YM, Kim HJ, Lee JS, et al. Hepatic angiomyolipoma with minimal fat, mimicking hepatocellular carcinoma. Clin Mol Hepatol.2012;18(3):330-335.[24] Akitake R, Kimura H, Sekoguchi S, Nakamura H, Seno H, Chiba T, et al. Perivascular epithelioid cell tumor (PEComa) of the liver diagnosed by contrast- enhanced ultrasonography. Intern Med. 2009;48(24):2083-6.

[25] Deng HY, Ding YY, Sun H, Liao CD, Xu W, Zhao S, et al. A variety of imaging manifestations of perivascular epithelioid cell tumor of liver. J Pract Med.2017;33 (13):2221-2225.

[26] Sun L, Sun X, Li Y, Xing L. The role of 18F-FDG PET/CT imaging in patient with malignant PEComa treated with mTOR inhibitor. Onco Targets Ther. 2015;30(8): 1967-1970.

[27] Touloumis Z, Giannakou N, Sioros C, Trigka A, Cheilakea M, Dimitriou N, et al. Retroperitoneal perivascular epithelioid cell tumours: a case report and review of literature. World J Clin Cases. 2019;7(21): 3524-3534.

[28] Kim SH, Kang TW, Lim K, Joh HS, Kang J, Sinn DH. A case of ruptured hepatic angiomyolipoma in a young male. Clin Mol Hepatol.2017;23(2):179-183. 
[29] Thway K, Fisher C. PEComa: morphology and genetics of a complex tumor family. Ann Diagn Pathol.2015;19(5):359-368.

[30] Folpe AL, Mentzel T, Lehr HA, Fisher C, Balzer BL, Weiss SW. Perivascular epithelioid cell neoplasms of soft tissue and gynecologic origin: a clinicopathologic study of 26 cases and review of the literature. Am J Surg Pathol.2005;29(12): 1558- 1575.

[31] Jafari A, Fischer HP, Von WM, Hong GS, Kalff JC, Manekeller S. Primary perivascular epithelioid cell tumor (PEComa) of the liver: case report and review of the literature. Z Gastroenterol.2013;51(09):10961100.

[32] Wang Q, Bai DS, Jiang GQ, Jin SJ. Perivascular epithelioid cell tumor of the liver: a case report. Chin J Gen Surg. 2016;31(11):973-974.

[33] Wang G, Gao J, Zhu JY, Leng XS, Hu ZP. Clinical study of perivascular epithelioid cell tumor of liver: a literature review of 41 cases. J Hepatobiliary Surg.2015;23(01): 10-15.

[34] Kirste S, Kayser G, Zipfel A, Grosu AL, Brunner T. Unresectable hepatic PEComa: a rare malignancy treated with stereotactic body radiation therapy (SBRT) followed by complete resection. Radiat Oncol. 2018;13(1):28-34.

[35] Wagner AJ, Malinowska-Kolodziej I, Morgan JA, Qin W, Fletcher CD, Vena N, et al. Clinical activity of mTOR inhibition with sirolimus in malignant perivascular epithelioid cell tumors: targeting the pathogenic activation of mTORC1 in tumors. J Clin Oncol.2010;28(5): 835-840.

\section{Tables}

Table 1 The sex, age, location, size, clinical symptoms and signs of 35 cases with hepatic perivascular epithelioid cell tumors 


\begin{tabular}{|c|c|c|c|c|c|c|c|}
\hline Number & Sex & $\begin{array}{l}\text { Age } \\
(\mathrm{yr})\end{array}$ & Location & $\begin{array}{l}\text { Size } \\
(\mathrm{cm})\end{array}$ & Symptoms & Signs & $\begin{array}{c}\text { Preoperative } \\
\text { diagnosis }\end{array}$ \\
\hline 1 & $\mathrm{~F}$ & 53 & S6/7 & 8 & None & None & HAML \\
\hline 2 & M & 25 & S6 & 11 & None & None & None \\
\hline 3 & $\mathrm{~F}$ & 62 & S1 & 2.5 & $\begin{array}{l}\text { Abdominal } \\
\text { pain }\end{array}$ & $\begin{array}{l}\text { Hepatic } \\
\text { tenderness }\end{array}$ & $\mathrm{HCC}$ \\
\hline 4 & $\mathrm{~F}$ & 58 & $\mathrm{~S} 6 / 7$ & 2.5 & None & None & $\mathrm{HCC}$ \\
\hline 5 & $\mathrm{~F}$ & 45 & $\mathrm{~S} 6 / 7$ & 4.5 & None & None & HAML \\
\hline 6 & $\mathrm{~F}$ & 54 & $\mathrm{~S} 2 / 3$ & 3.5 & $\begin{array}{l}\text { Abdominal } \\
\text { pain }\end{array}$ & $\begin{array}{l}\text { Hepatic } \\
\text { tenderness }\end{array}$ & HAML \\
\hline 7 & $\mathrm{~F}$ & 60 & $\mathrm{~S} 2 / 3$ & 7 & $\begin{array}{l}\text { Abdominal } \\
\text { pain }\end{array}$ & $\begin{array}{l}\text { Hepatic } \\
\text { tenderness }\end{array}$ & Lipoma \\
\hline 8 & M & 37 & S7 & 4 & None & None & None \\
\hline 9 & F & 59 & S5/8 & 1.5 & $\begin{array}{l}\text { Abdominal } \\
\text { pain }\end{array}$ & $\begin{array}{l}\text { Hepatic } \\
\text { tenderness }\end{array}$ & HCC \\
\hline 10 & $\mathrm{~F}$ & 37 & $\mathrm{~S} 6 / 7$ & 7 & $\begin{array}{l}\text { Abdominal } \\
\text { pain }\end{array}$ & $\begin{array}{l}\text { Hepatic } \\
\text { tenderness }\end{array}$ & HAML \\
\hline 11 & $\mathrm{~F}$ & 68 & $\mathrm{~S} 6 / 7$ & 2 & None & None & $\mathrm{HCC}$ \\
\hline 12 & F & 53 & S6 & 6 & $\begin{array}{l}\text { Abdominal } \\
\text { pain }\end{array}$ & None & None \\
\hline 13 & $\mathrm{~F}$ & 69 & S3 & 7 & None & $\begin{array}{l}\text { Hepatic } \\
\text { tenderness }\end{array}$ & None \\
\hline 14 & M & 37 & S5 & 3 & None & None & Hamartoma \\
\hline 15 & $\mathrm{~F}$ & 45 & S3 & 1.5 & $\begin{array}{l}\text { Abdominal } \\
\text { pain }\end{array}$ & None & $\begin{array}{c}\text { Hepatic } \\
\text { hemangioma }\end{array}$ \\
\hline 16 & $\mathrm{~F}$ & 51 & S8 & 9.5 & $\begin{array}{l}\text { Abdominal } \\
\text { pain }\end{array}$ & $\begin{array}{l}\text { Hepatic } \\
\text { tenderness }\end{array}$ & lipoma \\
\hline 17 & $\mathrm{~F}$ & 38 & S7 & 5 & None & None & None \\
\hline 18 & $\mathrm{~F}$ & 75 & S5 & 4 & None & None & $\mathrm{HCC}$ \\
\hline 19 & M & 21 & $\mathrm{~S} 2 / 3$ & 1.5 & None & None & $\mathrm{HCC}$ \\
\hline 20 & $\mathrm{~F}$ & 46 & S5 & 2 & $\begin{array}{l}\text { Abdominal } \\
\text { ge } 15 / 22\end{array}$ & Hepatic & HCC \\
\hline
\end{tabular}




\begin{tabular}{|c|c|c|c|c|c|c|c|}
\hline & & & & & pain & tenderness & \\
\hline 21 & $\mathrm{~F}$ & 52 & $\mathrm{~S} 2$ & 8 & $\begin{array}{l}\text { Abdominal } \\
\text { discomfort }\end{array}$ & $\begin{array}{c}\text { Hepatic } \\
\text { tenderness }\end{array}$ & None \\
\hline 22 & $\mathrm{~F}$ & 44 & $\mathrm{~S} 4$ & 3.5 & None & None & HAML \\
\hline 23 & $\mathrm{~F}$ & 50 & $\mathrm{~S} 4$ & 3 & None & None & HCC \\
\hline 24 & $\mathrm{~F}$ & 36 & S7 & $3.5 \square 2.5$ & & None & Hamartoma \\
\hline & & & & & None & & \\
\hline 25 & $\mathrm{~F}$ & 40 & S7 & 12 & None & None & HAML \\
\hline 26 & $\mathrm{~F}$ & 59 & $\begin{array}{l}\text { Right } \\
\text { lobe }\end{array}$ & $5 \square 3$ & $\begin{array}{l}\text { Abdominal } \\
\text { pain }\end{array}$ & None & Hamartoma \\
\hline 27 & $\mathrm{~F}$ & 21 & S6 & 7 & Abdominal & None & ICC \\
\hline 28 & $\mathrm{~F}$ & 49 & $\mathrm{~S} 5 / 8$ & 7.5 & $\begin{array}{l}\text { pain } \\
\text { Fever }\end{array}$ & None & $\mathrm{HCC}$ \\
\hline 29 & M & 60 & $\mathrm{~S} 4$ & 6.5 & $\underset{\text { pain }}{\text { Abdominal }}$ & None & None \\
\hline 30 & $\mathrm{~F}$ & 47 & $\mathrm{~S} 2 / 3$ & 3 & None & None & $\mathrm{HCC}$ \\
\hline 31 & M & 52 & S3 & 2.5 & None & None & $\begin{array}{l}\text { Hepatic } \\
\text { PEComa }\end{array}$ \\
\hline 32 & $\mathrm{~F}$ & 28 & $\begin{array}{l}\text { Right } \\
\text { lobe }\end{array}$ & 8 & None & None & None \\
\hline 33 & $\mathrm{~F}$ & 43 & $\mathrm{~S} 1$ & 1.2 & None & None & Hamartoma \\
\hline 34 & $\mathrm{~F}$ & 39 & $\mathrm{~S} 4$ & 4 & None & None & None \\
\hline 35 & $\mathrm{~F}$ & 38 & S4 & 4 & None & None & HAML \\
\hline
\end{tabular}

HCC: Hepatocellular carcinoma; HAML: Hepatic angiomyolipoma; PEComa: perivascular epithelioid cell tumor; ICC: Intrahepatic cholangiocarcinoma. 
Table 2 The pathological features of 35 cases with hepatic perivascular epithelioid cell tumors 


\begin{tabular}{|c|c|c|c|c|c|c|}
\hline Number & Texture & Growth pattern & Boundary & Colour & Capsule & Adipose tissues \\
\hline 1 & Soft & Exophytic & Clear & Yellow & + & + \\
\hline 2 & Soft & Exophytic & Clear & Red & - & + \\
\hline 3 & Moderate & Exophytic & Clear & Yellow & - & - \\
\hline 4 & Soft & Exophytic & Clear & Yellow & - & - \\
\hline 5 & Soft & Exophytic & Clear & Yellow & - & + \\
\hline 6 & Soft & Exophytic & Clear & Yellow & - & + \\
\hline 7 & Soft & Exophytic & Clear & Yellow & + & + \\
\hline 8 & Moderate & Exophytic & Clear & Yellow & - & + \\
\hline 9 & Soft & Exophytic & Clear & Yellow & + & + \\
\hline 10 & Soft & Exophytic & Clear & Off-white & - & + \\
\hline 11 & Soft & Exophytic & Clear & Yellow & + & + \\
\hline 12 & Soft & Exophytic & Clear & Yellow & - & + \\
\hline 13 & Soft & Exophytic & Clear & Red & - & + \\
\hline 14 & Soft & Exophytic & Clear & Yellow & + & + \\
\hline 15 & Moderate & Exophytic & Clear & Yellow & + & + \\
\hline 16 & Soft & Exophytic & Clear & Yellow & - & + \\
\hline 17 & Hard & Exophytic & Unclear & $\begin{array}{l}\text { Pinkish- } \\
\text { grey }\end{array}$ & - & + \\
\hline 18 & Soft & Exophytic & Unclear & Red & - & + \\
\hline 19 & Soft & Exophytic & Clear & Yellow & - & + \\
\hline 20 & Soft & Exophytic & Clear & Yellow & - & + \\
\hline 21 & Moderate & Exophytic & Clear & Red & - & + \\
\hline 22 & Soft & Exophytic & Clear & Yellow & - & + \\
\hline 23 & Soft & Exophytic & Clear & Yellow & - & + \\
\hline 24 & Soft & Exophytic & Clear & Yellow & - & + \\
\hline 25 & Soft & Exophytic & Clear & Red & + & + \\
\hline 26 & Soft & Exophytic & Clear & Yellow & - & + \\
\hline 27 & Soft & Exophytic & Clear & Yellow & - & + \\
\hline
\end{tabular}




\begin{tabular}{llllll}
28 & Hard & Infiltrative & Unclear & Yellow & + \\
29 & Soft & Exophytic & Clear & Yellow & + \\
30 & Soft & Exophytic & Clear & Yellow & + \\
31 & Soft & Exophytic & Clear & Yellow & + \\
32 & Soft & Exophytic & Clear & Yellow & + \\
33 & Soft & Exophytic & Clear & Yellow & + \\
34 & Soft & Exophytic & Clear & Yellow & + \\
35 & Hard & Exophytic & Clear & Off-white & + \\
\hline
\end{tabular}

\section{Figures}

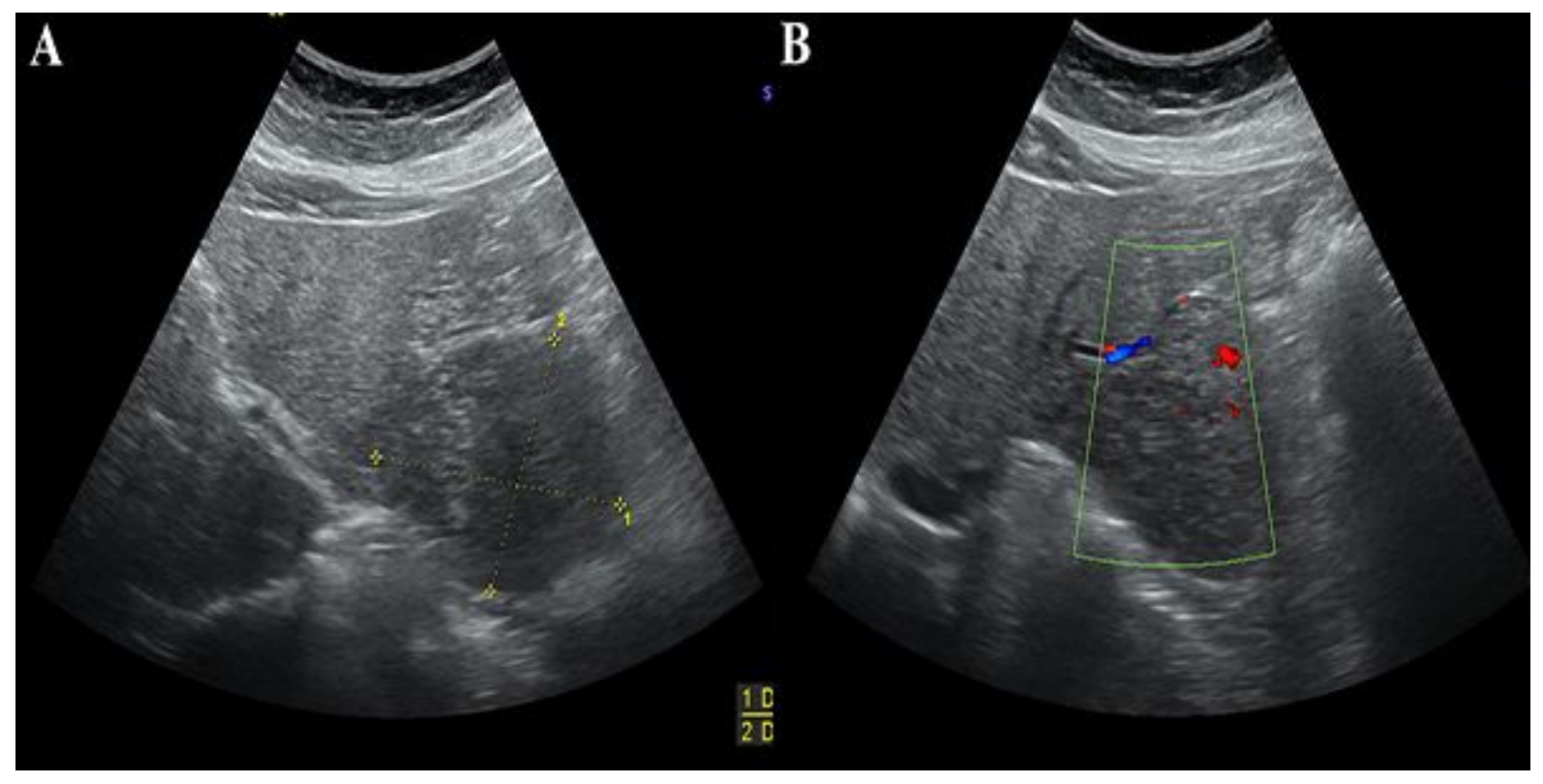

\section{Figure 1}

Ultrasound image of a hepatic PEComa. (a) Ultrasound showed a heterogeneous mass with a low echogenicity. (b) CDFI showed blood flow signals in the mass. CDFI= colour Doppler flow imaging. 

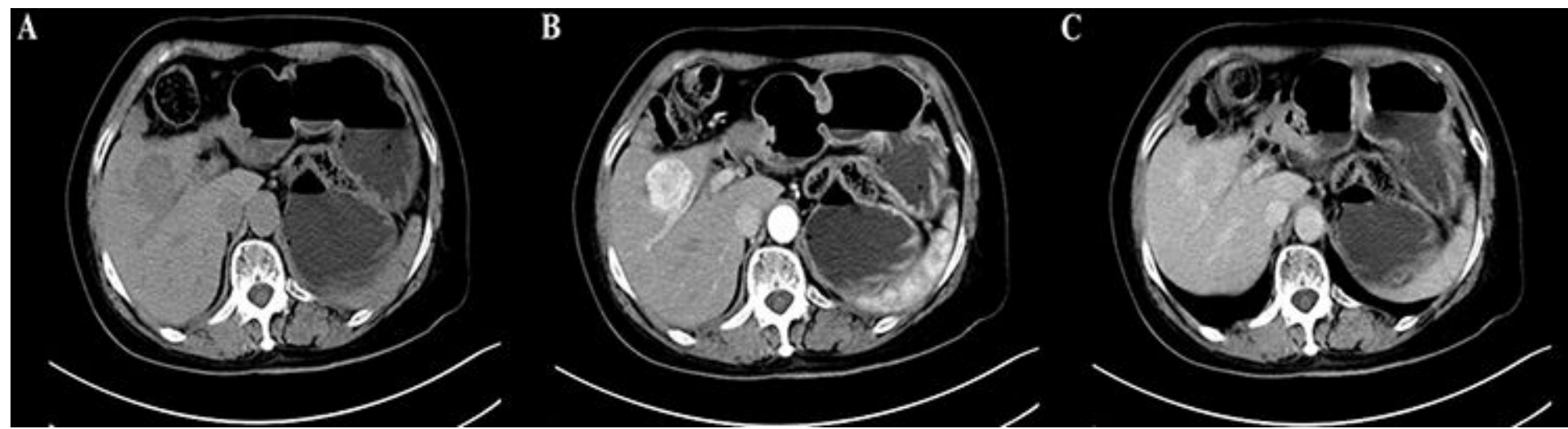

Figure 2

CT images of hepatic PEComas. (a) The plain CT scan revealed a quasi-circular mass with an inhomogeneous low density and clear boundaries. (b) The contrast-enhanced CT scan showed the obvious heterogeneous enhancement of the same lesion in the atrial phase. (c) The contrast-enhanced CT scan showed a marked weakening of the enhancement of the same lesion. CT= computed tomography.

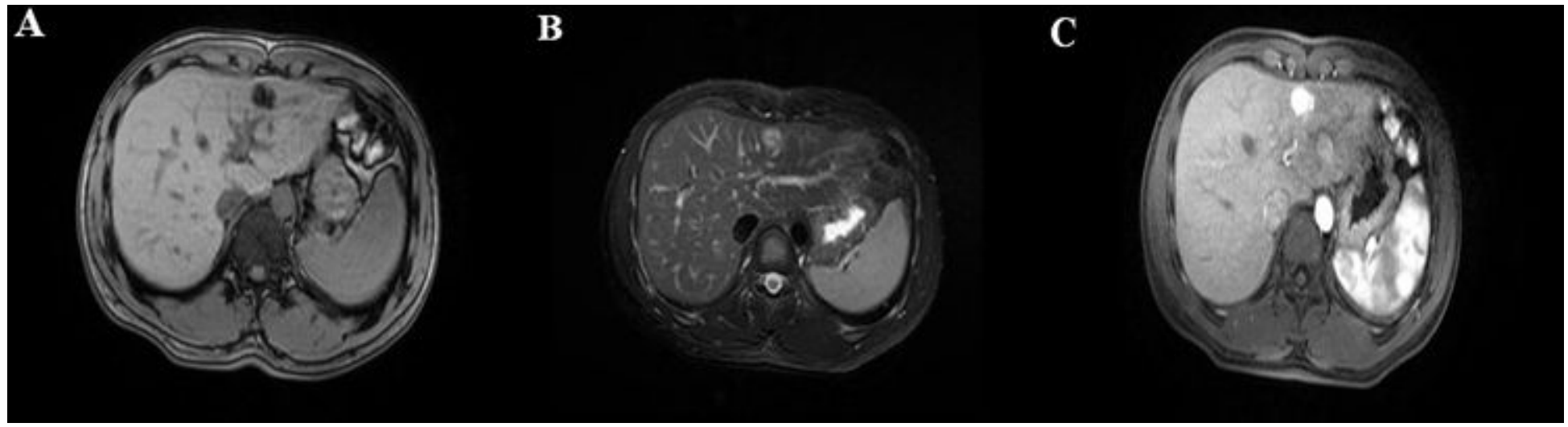

\section{Figure 3}

MRI of hepatic PEComas. (a) MRI revealed a tumor characterized by a heterogeneous hypointensity on T1-weighted images of the left lobe. (b) The mass displayed heterogeneous hyperintensity on T2weighted images. (c) The lesion was significantly enhanced on diffusion-weighted imaged. MRI= magnetic resonance imaging. 


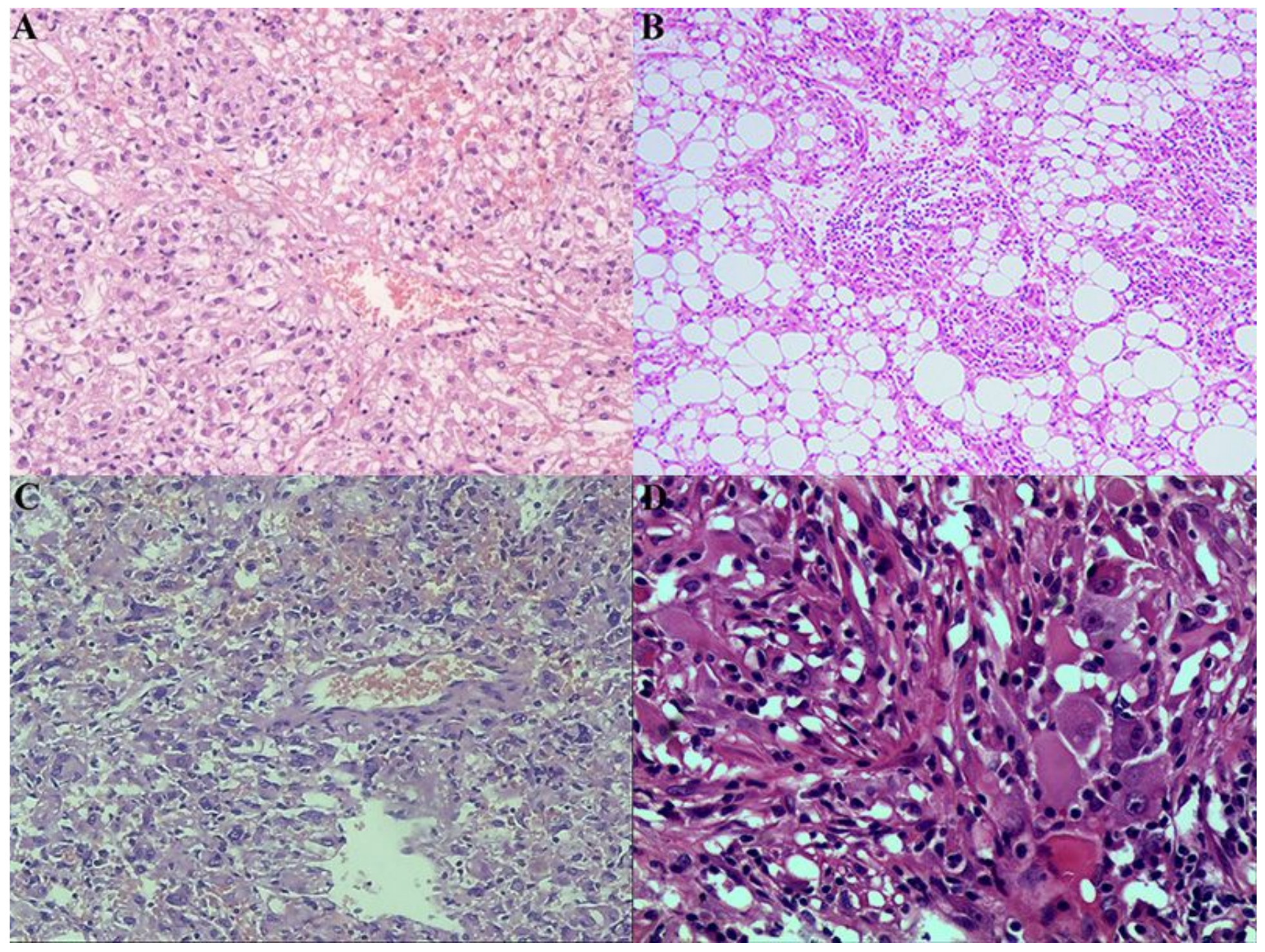

\section{Figure 4}

Microscopic pathological appearances of hepatic PEComas. (a) Epithelioid neoplastic cells were arranged radially around blood vessels (H\&E staining, 100x). (b) Fat, smooth muscles and blood vessels (H\&E staining, 100x). (c) Thick-walled vessels and dilated hepatic sinusoids (H\&E staining, 100x). (d) Megakaryocytes and cells with atypical nuclei (H\&E staining, 200x). H\&E= haematoxylin and eosin.

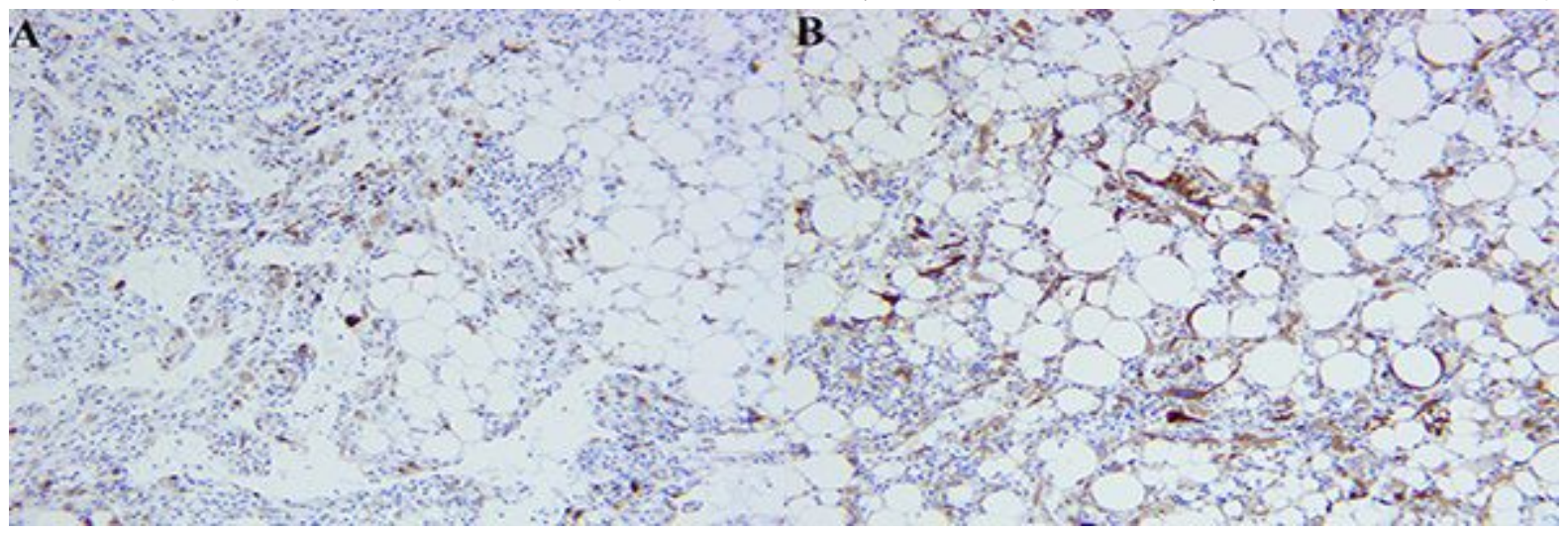


Immunohistochemical characteristics of hepatic PEComas. (a) Human melanoma black-45 staining (EnVision method, 100x). (b) Melan-A staining (EnVision method, 100x). 